\title{
Locally advanced gastric cancer: total iodine uptake to predict the response of primary lesion to neoadjuvant chemotherapy
}

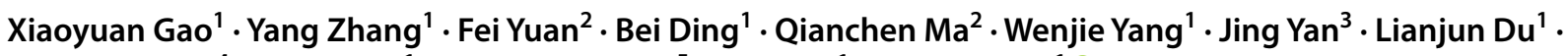 \\ Baisong Wang ${ }^{4} \cdot$ Fuhua Yan $^{1} \cdot$ Martin Sedlmair $^{5} \cdot$ Zilai Pan $^{1} \cdot$ Huan Zhang ${ }^{1}$ (i)
}

Received: 23 June 2018 / Accepted: 30 July 2018 / Published online: 9 August 2018

(c) The Author(s) 2018

\begin{abstract}
Purpose Pathologic response to neoadjuvant chemotherapy is a prognostic factor in many cancer types. However, the existing evaluative criteria are deficient. We sought to prospectively evaluate the total iodine uptake derived from dual-energy computed tomography (DECT) in predicting treatment efficacy and progression-free survival (PFS) time in gastric cancer after neoadjuvant chemotherapy.

Methods From October 2012 to December 2015, 44 patients with locally advanced gastric cancer were examined with DECT 1 week before and three cycles after neoadjuvant chemotherapy. The percentage changes in tumor area $(\% \Delta S)$, diameter $(\% \Delta D)$, and density $(\% \Delta \mathrm{HU})$ were calculated to evaluate the WHO, RESCIST, and Choi criteria. The percentage changes in tumor volume $(\% \Delta V)$ and total iodine uptake of portal phase (\% $\%$ TIU-p) were also calculated to determine cut-off values by ROC curves. The correlation between the different criteria and histopathologic tumor regression grade (Becker score) or PFS were statistically analyzed.

Results Forty-four patients were divided into responders and non-responders according to $43.34 \%$ volume reduction $(P=0.002)$ and $63.87 \%(P=0.002)$ TIU-p reduction, respectively. The \% $\Delta$ TIU-p showed strong $(r=0.602, P=0.000)$ and $\% \Delta V$ showed moderate $(r=0.416, P=0.005)$, while the WHO $(r=0.075, P=0.627)$, RECIST $(r=0.270, P=0.077)$ and Choi criteria $(r=0.238, P=0.120)$ showed no correlation with the Becker score. The differences in PFS time between the responder and non-responder groups were significant according to \% $\Delta$ TIU-p and Choi criteria $(P=0.001$ and $P=0.013$, respectively). Conclusions The TIU-p can help predict pathological regression in advanced gastric cancer patients after neoadjuvant chemotherapy. In addition, the $\% \Delta$ TIU-p could be one of the potentially valuable predictive parameters of the PFS time.
\end{abstract}

Keywords Gastric cancer $\cdot$ Neoadjuvant chemotherapy $\cdot$ Total iodine uptake $\cdot$ Histopathologic regression $\cdot$ Progression-free survival (PFS)

\section{Abbreviations}

TIU Total iodine uptake

TIU-p Total iodine uptake of portal phase

Xiaoyuan Gao and Yang Zhang have contributed equally to this work.

Zilai Pan

zilaipanlilly@163.com

$\triangle$ Huan Zhang

huanzhangy@163.com

1 Department of Radiology, Ruijin Hospital, Shanghai Jiao Tong University School of Medicine, No. 197, Ruijin 2nd Road, Shanghai 200025, China

2 Department of Pathology, Ruijin Hospital, Shanghai Jiao Tong University School of Medicine, No. 197, Ruijin 2nd Road, Shanghai 200025, China
$S \quad$ Largest tumor area of the axial images

$D \quad$ Longest tumor diameter of the axial images

HU Tumor mean density

$V \quad$ Tumor volume

PFS Progression-free survival

3 Siemens Medical System, Shanghai 201318, China

4 Department of Biological Statistics, Shanghai Jiao Tong University School of Medicine, Shanghai 200025, China

5 Computed Tomography Research and Development, Siemens Healthcare GmbH, Forchheim, Germany 
$\% \Delta S \quad$ Percentage change in the tumor area

$\% \Delta D \quad$ Percentage change in the tumor diameter

$\% \Delta \mathrm{HU} \quad$ Percentage change in the tumor density

$\% \Delta V \quad$ Percentage change in the tumor volume

$\% \Delta$ TIU-p Percentage change in the total iodine uptake of the tumor in portal phase

\section{Introduction}

In patients with potentially operable gastric cancer, neoadjuvant chemotherapy decreased tumor size and stage to significantly improve progression-free and overall survival, as established in several randomized Phase III studies (Putter 2006; Ychou et al. 2011). Published data indicated that patients who pathologically respond to neoadjuvant chemotherapy have a better prognosis after surgery than patients who do not respond to chemotherapy (Bichev et al. 2015). Because some patients with gastric cancer are insensitive to chemotherapeutics, the evaluative criteria of iconography to predict the effect of neoadjuvant chemotherapy are very important.

Traditionally, the WHO and RECIST criteria have been used to evaluate therapeutic response based on reductions in tumor area (product of longest diameter and the perpendicular diameter) and longest tumor diameter, respectively (Eisenhauer et al. 2009; Staquet 1981). However, stomach is generally not considered as a suitable target due to its unfixed shape, which prevents the stable and reproducible measurement of the longest tumor diameter (Ott et al. 2003). Currently, tumor volumetry is used to predict the response of gastric cancer to treatment (Sang et al. 2009), and the threedimensional (3D) data have been used to evaluate morphological changes. However, previous studies have suggested that the change in tumor size is not proportional to the number of tumor cells; tumors might show changes in internal components and small decreases in size, especially in targeted therapy (Benjamin et al. 2007; Motzer et al. 2006).

Accordingly, the Choi criteria (combining the change in the sum of the largest tumor diameter with the change in the tumor density) have been used as a potential indicator of the GIST (gastrointestinal stromal tumor) response in patients undergoing targeted therapy to add functional information to size-based monitoring (Choi 2008). Although ${ }^{18}$ F-FDG$\mathrm{PET} / \mathrm{CT}$ has been shown to be able to assess the response of gastric cancer to neoadjuvant chemotherapy based on the change in the ${ }^{18}$ F-FDG standardized uptake value (Giganti et al. 2015), signet-ring cell carcinomas, mucinous adenocarcinomas and poorly differentiated adenocarcinomas exhibit relatively low ${ }^{18} \mathrm{~F}$-FDG uptake (Chen et al. 2016; Lee et al. 2015), which may prevent the use of ${ }^{18} \mathrm{~F}$-FDG-PET/ $\mathrm{CT}$ to monitor tumor response. Furthermore, this imaging modality is associated with prohibitively high costs.
Dual-energy CT (DECT) allows the differences in spectral absorption behavior between materials of different elemental composition to be exploited. This method uses two acquisitions with X-ray spectra that differ in their mean photon energies to gain additional information on the scanned material. Based on the complementary information from the two acquisitions at low and high tube potential, 3-material decomposition can subsequently be performed to quantify the concentrations of elements within the human body (Stiller et al. 2015). With the injection of an iodinated contrast agent, qualitative and quantitative DECT iodine concentration maps can provide an approximation of tissue physiology (Stiller et al. 2015; Yeh et al. 2009). Iodine uptake (IU), which is derived from the iodine concentration map, has been used as a quantitative perfusion parameter in the myocardium (Sánchez-Gracián et al. 2015). In addition, IU may be regarded as a functional parameter or a more robust response parameter to evaluate the response of the tumor to therapy by reflecting the accumulation of contrast medium (Apfaltrer et al. 2012; Uhrig et al. 2013). However, the knowledge learned from the prior preliminary studies with HCC (Apfaltrer et al. 2012) and GIST (Dai et al. 2013) could not be transferred to gastric cancer directly. In addition, few studies have addressed the use of the total IU (TIU) in the tumor for the evaluation of the efficacy of neoadjuvant therapy in patients with locally advanced gastric cancer.

This study aimed to prospectively evaluate the total iodine uptake derived from DECT in the assessment of treatment efficacy and for the prediction of the progression-free survival (PFS) time in patients with locally advanced gastric cancer after neoadjuvant chemotherapy.

\section{Materials and methods}

\section{Patients}

The prospective study protocol was approved by our local ethics committee. From October 2012 to December 2015, 65 patients who met the inclusion criteria were enrolled in this study (Fig. 1). Written informed consent was obtained from all patients.

The inclusion criteria were as follows: (a) a preliminary pathological diagnosis of gastric carcinoma based on a gastroscopic biopsy without previous chemotherapy; (b) a tumor defined as locally advanced $\left(\mathrm{cT}_{3 \sim 4 b} \mathrm{~N}_{1 \sim 3} \mathrm{M}_{0}\right)$ gastric cancer based on CT examinations; (c) a WHO performance status of $\leq 2$, an age of 18-75 years, blood count, normal liver and renal function in normal range, a grade $<2$ for peripheral neuropathy, and no distant metastases; and (d) standard neoadjuvant chemotherapy and radical resection.

Twenty-one patients were excluded, and the exclusion criteria were as follows: (a) did not undergo preoperative 
Fig. 1 Flowchart of the study population
Inclusion criteria:

(a) a preliminary pathological diagnosis of gastric carcinoma based on a gastroscopic biopsy without previous chemotherapy;

(b) a tumor defined as locally advanced $\left(\mathrm{cT}_{3-4 b} \mathrm{~N}_{1-3} \mathrm{M}_{0}\right)$ gastric cancer based on CT examinations:

(c) a WHO performance status of $=2$, an age of 18-75 years, blood counts, normal liver and renal function in normal range, a grade $<2$ for peripheral neuropathy, and no distant metastases:

(d) standard neoadjuvant chemotherapy and radical resection.

\begin{tabular}{|l}
21 patients were excluded: \\
(a) did not undergo preoperative treatment or receive the full course of planned \\
postoperative chemotherapy because of poor performance status, complications \\
or compliance issues in the postoperative period (n=11): \\
(b) did not undergo dual-energy enhanced CT exam inations 1 week before or \\
three cycles after the start of chemotherapy (n=5): \\
(c) poor image quality, such as from poor gastric lumen filling ( $\mathrm{n}=3$ ); \\
(d) peritoneal metastasis was found during exploratory operation (n=2).
\end{tabular}

treatment or receive the full course of planned postoperative chemotherapy because of poor performance status, complications or compliance issues in the postoperative period ( $n=11$ ); (b) did not undergo dual-energy enhanced CT examinations 1 week before or three cycles after the start of chemotherapy $(n=5)$; (c) poor image quality, such as from poor gastric lumen filling $(n=3)$; and (d) peritoneal metastasis was found during exploratory operation $(n=2)$.

\section{Neoadjuvant chemotherapy}

The patients were treated with epirubicin $\left(50 \mathrm{mg} / \mathrm{m}^{2}\right.$ on day 1 , every 3 weeks $)$, cisplatin $\left(60 \mathrm{mg} / \mathrm{m}^{2}\right.$ on day 1 , every 3 weeks $)$ and $5-\mathrm{FU}\left(200 \mathrm{mg} / \mathrm{m}^{2}\right.$ on days $1-21$ through an ambulatory infusion pump, every 3 weeks). According to the MAGIC protocol, three preoperative and three postoperative cycles were planned (Putter 2006).

\section{CT protocol}

All patients received DECT examinations 1 week before (baseline examination) and three cycles after (follow-up examination) the start of chemotherapy. The scanning protocol was based on previous research (Shi et al. 2016). The patients underwent CT after overnight fasting to empty the stomach. Before the CT examinations, each patient drank $1000-1500 \mathrm{ml}$ of water and was injected with a hypotonic agent (20 $\mathrm{mg}$ of scopolamine); then, they underwent a scan with a second-generation DECT scanner (Siemens SOMATOM Definition Flash; Siemens Medical Solutions, Forchheim, Germany). The CT scans were acquired with the tube voltages at $100 / \mathrm{Sn} 140 \mathrm{kV}$ with a tin filter using references of 230 and $178 \mathrm{mAs}$. The collimator was $128 \times 0.6 \mathrm{~mm}$. All acquisitions were obtained in real-time by the automatic dose modulation protocol CareDose 4D (Siemens Healthcare, Forchheim, Germany). Sixteen milliliters of contrast agent were first injected as a test bolus 
to estimate the time to the peak enhancement of the celiac trunk. The main bolus ( $1.5 \mathrm{ml}$ iopromide per kilogram of body weight, Ultravist 370; Schering, Berlin, Germany) was then injected at a rate of $3 \mathrm{ml} / \mathrm{s}$. Two-phase contrastenhanced DECT scans were performed, and these scans included the arterial phase (at the beginning of the peak enhancement of the celiac trunk), which covered the entire stomach, and the portal phase (20 s after the peak enhancement of the celiac trunk), which ranged from the diaphragmatic domes to the anal verge. The mean delay time of the portal phase was $39.06 \pm 7.644 \mathrm{~s}$ (range 30-51 s after injection). All DICOM data were transferred to the workstation and interpreted by the radiologists using a novel software prototype ("Dual Energy Tumor Evaluation" running on a prototype platform eXamine, 0.9.3.0, Siemens Healthcare, Forchheim, Germany), which was used only for research purposes at our institution.

The total volume CT dose index (CTDIvol) and doselength product (DLP) of the baseline and follow-up examinations for each patient were obtained from the dose report on the CT scanner. The size-specific dose estimate (SSDE) of the baseline and follow-up examinations was calculated based on the individual effective patient diameter, as measured from the axial CT images (Christner et al. 2013).

\section{Image analysis}

The DECT images were reconstructed with FBP to obtain a $1.0 \mathrm{~mm}$ slice thickness with a $1.0 \mathrm{~mm}$ increment and evaluated separately by two radiologists (Du and Pan), who had more than 10 years of gastrointestinal diagnostic experience and were completely blinded to the clinical information of the patients, the results of surgery and the results of the histopathologic examination (they were aware that the patients had histologically proven gastric cancers, but they were completely blinded to lesion location, size, stage and treatment of the gastric cancers). Before the actual reading, a training session of 10 scans for the volumetric measurements was provided to enable the readers to familiarize themselves with the software and share their experiences. The ICC was measured and proved to be approximately 0.9 ; therefore, we regarded the two radiologists as qualified to perform reliable measurements for our following study. Thus, similar criteria for defining the tumor boundary could be established. Primary lesions were evaluated at the baseline and follow-up CT examinations. Additionally, the mean value of the two readers' measurements was used for further analysis.

The two radiologists interpreted one CT scan each time without knowing if it was pretreatment or posttreatment. All images were anonymized. The largest tumor area $(S)$ and longest diameter $(D)$ of the axial images, the tumor density $(\mathrm{HU})$, the volume $(V)$ and total iodine uptake of the portal phase (TIU-p, mg) were measured by a semiautomatic tumor segmentation algorithm (Grady 2006; Uhrig et al. 2013). This algorithm calculated the semiautomatic 3D segmentation and volume of interest (VOI) based quantification of iodine uptake after the user had drawn an approximate diameter of the primary lesion on a representative axial slice. After indicating the transverse diameter of a lesion, VOIs were automatically calculated and used to quantify iodine uptake across the entire lesion. The manual refinement of the VOI margin was undertaken by the radiologists according to their recognition of the primary lesions in some axial slices for lesion margins that were not satisfactorily contoured by the software (Fig. 2). The contrast medium enhancement was quantified based on three material decompositions, assuming the main components were fat, soft tissue and iodine (Uhrig et al. 2013). On the basis of calibration measurements performed by the manufacturer, the algorithm is capable of transforming spectral information of dual-energy data into absolute values of iodine uptake. In addition to quantification, the software also provides visualization of IU by color-coding the amount of IU in each voxel and displaying the results in a two-dimensional overview (Fig. 2c, f). The relative percentage changes in $S, D, \mathrm{HU}, V$ and TIU-p after neoadjuvant chemotherapy were calculated by another radiologist (B Ding) using the following formula:

$\% \Delta S=(S$-after $-S$-before $) / S$-before $\times 100 \%$,

$\% \Delta D=(D$-after $-D$-before $) / D$-before $\times 100 \%$,

$\% \Delta \mathrm{HU}=(\mathrm{HU}-$ after $-\mathrm{HU}$-before $) /$ HU-before $\times 100 \%$,

$\% \Delta V=(V$-after $-V$-before $) / V$-before $\times 100 \%$,

$\% \Delta \mathrm{TIU}-\mathrm{p}=(\mathrm{TIU}-\mathrm{p}$-after $-\mathrm{TIU}$-p-before $) /(\mathrm{TIU}-\mathrm{p}$-before $) \times 100 \%$.

According to the WHO, RECIST and Choi criteria, patients exhibiting a complete response (CR) and partial response (PR) were classified into the responder group, whereas patients with stable disease (SD) and progressive disease (PD) were classified into the non-responder group. The cut-off criteria for partial response (PR) by WHO, RECIST were $50 \%$ and $30 \%$. In addition, in Choi criteria, a decrease in size of $\geq 10 \%$ or a decrease in tumor density (HU) $\geq 15 \%$ on CT is regarded as PR (Eisenhauer et al. 2009).

\section{Surgery}

Patients were transferred to surgery 3 weeks after the last cycle of chemotherapy. The acceptable resections included a radical total gastrectomy and a subtotal distal gastrectomy. In both procedures, the resection lines were required to be at least $3 \mathrm{~cm}$ from the edge of the macroscopic tumor. The recommended surgical procedure is a D2 gastrectomy to 
Fig. 2 Baseline CT images (a-c) and follow-up CT images (d-f) of a patient (Female,

73 years) after three cycles of chemotherapy; $\mathbf{a}, \mathbf{d}$ were mixed energy images of portal phase; b, e were corresponding iodine maps; right figures in $\mathbf{c}, \mathbf{f}$ were the distribution of density values of the identified lesion in the iodine maps, mixed and virtual non-contrasted (VNC) images. The target lesion was semiautomatic segmented in 3D and with color-labeled tumor displayed on a representative axial slice, as shown in left in $\mathrm{c}$ and $\mathrm{f}$ (blue parts in tumor), ( $\% \Delta S:-43.49 \%$, $\% \Delta D:-15.92 \%, \% \Delta \mathrm{HU}$ : $-15.88 \%, \% \Delta V:-76.13 \%$, $\% \Delta$ TIU-p: $-78.93 \%$ for this patient). According to the WHO, RECIST criteria, this patient was classified as a non-responder, but according to other criteria, he was classified as a responder. The pathology of this patient $(\mathrm{g})$ was grade 1 , complete ( $0 \%$ residual tumor) tumor regression. Based on the definite diagnosis, this patient had survived 36 months and survives to this day
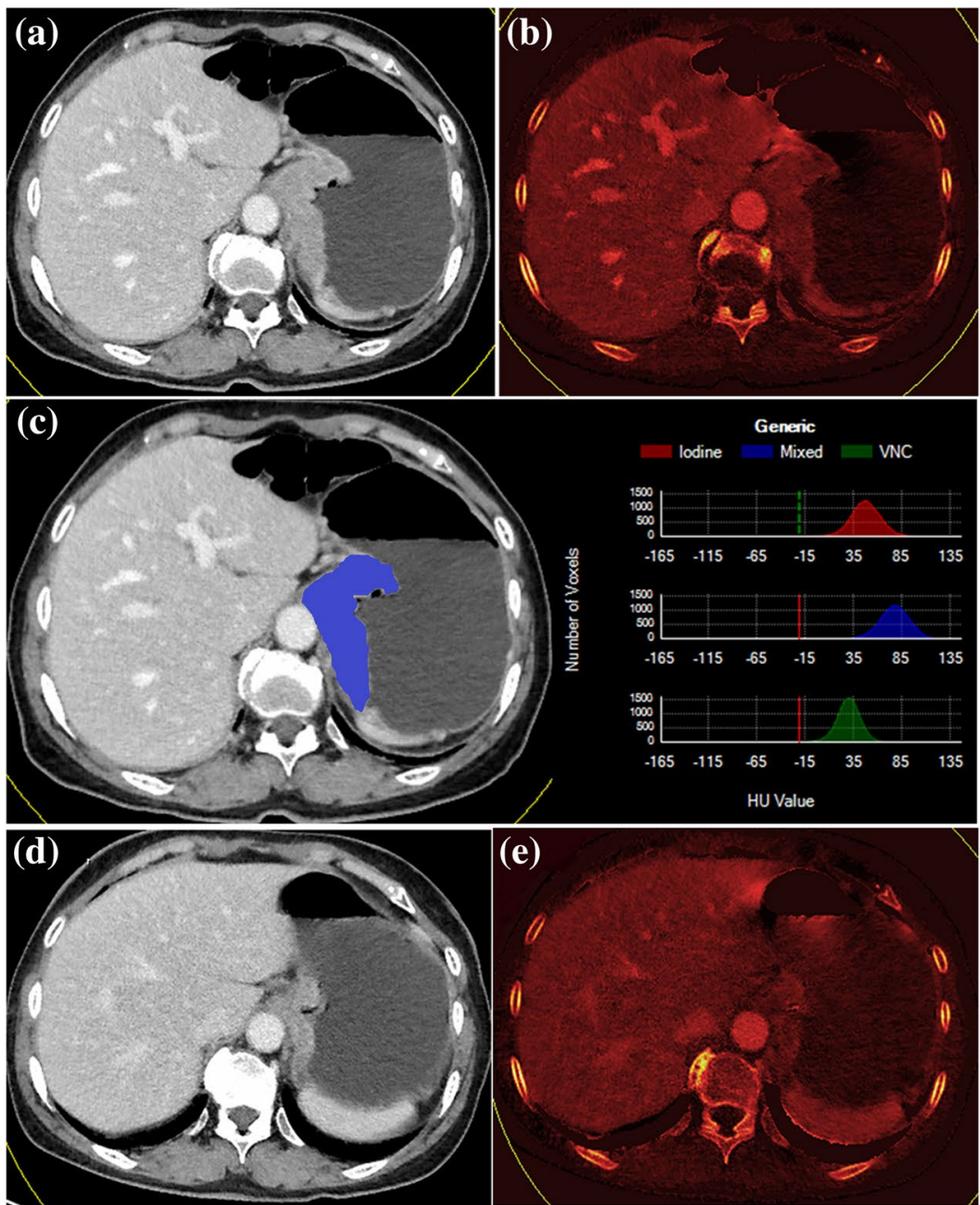

(e)

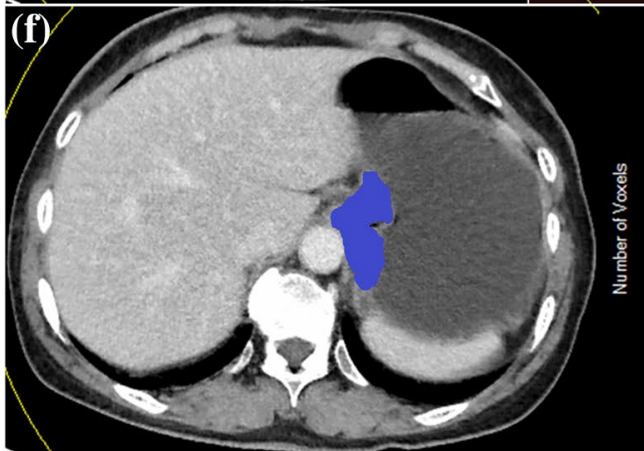

$\infty$
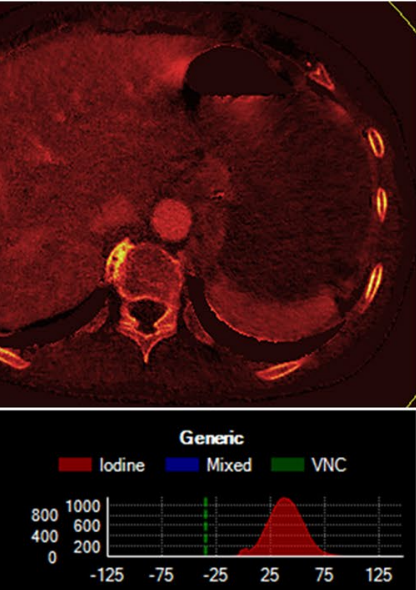

$\begin{array}{rr}800 & 1000 \\ 400 & 600 \\ 0 & 200\end{array}$
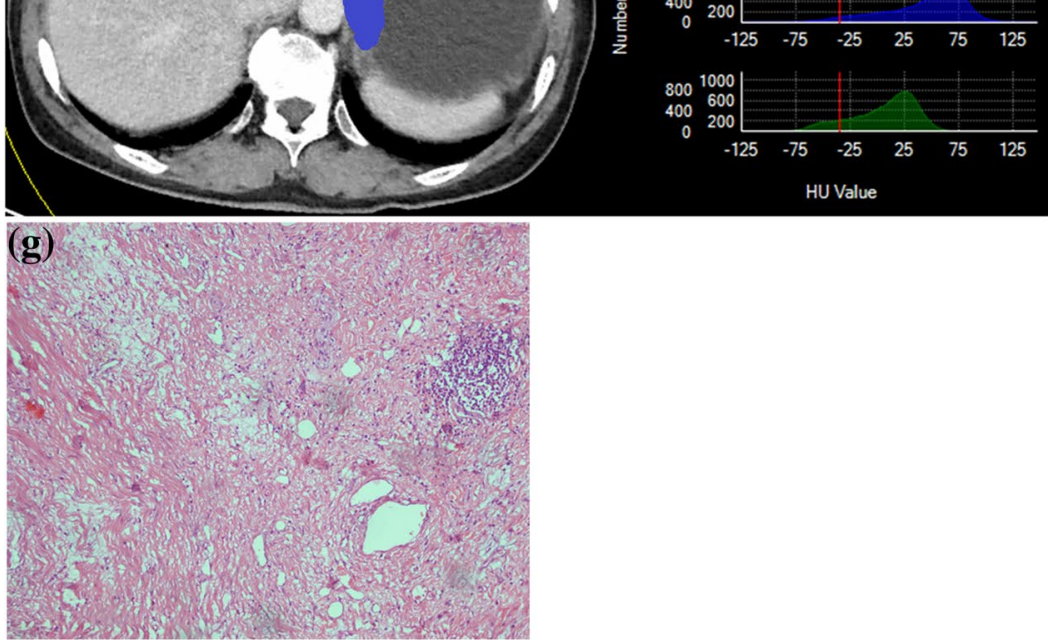
ensure the complete resection of the primary cancer and its draining nodes.

\section{Histopathologic analysis}

Two pathologists with 10 years of experience in histopathologic response evaluation carefully reviewed all the original resected specimens from the patients until a consensus was reached. The macroscopically identifiable residual tumor was measured. The entire macroscopically identifiable tumor or the area of the stomach with scarring, which indicates the site of the previous tumor (the tumor bed), was serially cross-sectioned at $0.5 \mathrm{~cm}$ intervals. All slides were stained with hematoxylin and eosin and elastic van Gieson stain. The van Gieson stain was used to distinguish between tumor desmoplasia and scarring as a result of chemotherapy. The Becker score, which is based on an estimation of the percentage of vital tumor tissue, was used to evaluate the histopathologic tumor regression grade. The following three grades were used: grade 1 , complete $(0 \%$ residual tumor) or subtotal tumor regression $(<10 \%$ residual tumor per tumor bed); grade 2, partial tumor regression (10-50\% residual tumor per tumor bed); and grade 3, minimal or no tumor regression ( $>50 \%$ residual tumor per tumor bed)(Becker et al. 2003). The patients with grade 1 were categorized as responders while patients with grade 2 or 3 were categorized as non-responders.

\section{Patient follow-up}

After discharge, all patients were followed-up with physical and blood examinations every 4-12 weeks; in addition, chest X-rays and abdominal and pelvic CT scans were performed every 3 months for the first year. Follow-ups were conducted at 6-month intervals during the second year. PFS was defined as the time from diagnosis to local recurrence, distant metastasis, or death or the last follow-up date for patients without local recurrence, distant metastasis or death.

\section{Statistical analysis}

Intraclass correlation coefficients (ICC) were exploited to test the inter-rater reliability of the measurement between the two readers. An ICC greater than 0.75 indicated good agreement (Landis and Koch 1977). The distribution of different parameters in different histopathology was described by a boxplot.

Receiver operating characteristic (ROC) curves were employed to evaluate the diagnostic accuracy of different indicators to preoperatively predict the histopathologic regression of gastric cancer after neoadjuvant chemotherapy and to determine cut-off values that maximized the sum of the sensitivity and specificity. The area under the ROC curve (AUC) was determined with the corresponding 95\% confidence interval. The correlations between the WHO, RECIST, Choi criteria, $\% \Delta V, \% \Delta$ TIU-p and the histopathologic response were analyzed by Spearman rank correlation. The correlation is considered very weak, weak, moderate, strong or very strong when the correlation coefficient $(r)$ is $\leq 0.199,0.200-0.399,0.400-0.599,0.600-0.799$, and $\geq 0.800$, respectively. The association between different groups (responders and non-responders according to the cut-off value of the WHO, RECIST, Choi criteria, $\% \Delta \mathrm{V}$ and $\% \Delta \mathrm{TIU}-\mathrm{p}$ ) and PFS time were assessed with a log-rank test, and the survival rates were estimated using a Kaplan-Meier curve. All statistical tests were performed at a 5\% significance level using the SPSS software (version 17.0; SPSS, Inc., Chicago, IL).

\section{Results}

Forty-four patients were included in the final analysis $(n=44,16$ females and 28 males; the mean age \pm standard deviation, 58 years \pm 8.8 ). By location, 17 tumors occurred on the body, 12 on the antrum, 4 on the fundus, 3 on the cardia, 6 on the body-antrum and 2 on the cardia-body. Twenty-eight of 44 patients underwent a total gastrectomy, and 16 patients underwent a subtotal gastrectomy. According to the histopathological regression, 14 of 44 patients were classified as grade 1, 18 patients were classified as grade 2 , and 12 patients were classified as grade 3 . Six patients were found to have intratumoral hemorrhage proved by the pathology.

\section{Tumor change indicators and histopathologic regression after neoadjuvant chemotherapy}

The inter-rater reliability of the measurement between the two readers was significantly consistent (ICC $>0.75$, Table 1).

The distribution of different parameters in different histopathologies is shown in Fig. 3a.

The AUC of WHO, RECIST, Choi criteria, $\% \Delta V$, and $\% \Delta$ TIU-p were 0.495 (95\% CI 0.310-0.680), 0.617 (95\% CI $0.433-0.800$ ), 0.681 (95\% CI 0.521-0.840), 0.788 (95\% CI $0.652-0.924$ ), and 0.786 (95\% CI 0.648-0.923), respectively (Table 2; Fig. 3b, c). Only $\% \Delta V$ and $\% \Delta$ TIU-p had significant predictive ability $(P<0.01)$; therefore, a decreased rate $\geq 43.34 \%$ for $\% \Delta V$ or $\geq 63.87 \%$ for $\% \Delta$ TIU-p can be regarded as the positive responders (Table 2; Fig. 3c).

The stratification of patients by histopathologic regression grade is shown in the Table 3 , according to the different groups of WHO, RECIST, Choi criteria, $\% \Delta V$, and $\% \Delta$ TIU-p. Spearman correlation demonstrated that the groups of $\% \Delta$ TIU-p strongly correlated with the Becker 
Table 1 The inter-rater reliability of the measurement between the two readers

\begin{tabular}{llllll}
\hline & $S_{\text {before }}$ & $S_{\text {after }}$ & $D_{\text {before }}$ & $D_{\text {after }}$ & HU $_{\text {before }}$ \\
\hline ICC & 0.788 & 0.803 & 0.834 & 0.840 & 0.876 \\
\hline & $\mathrm{HU}_{\text {after }}$ & $V_{\text {before }}$ & $V_{\text {after }}$ & TIU-p & before \\
\hline ICC & 0.895 & 0.894 & 0.885 & 0.928 & 0.916 \\
\hline
\end{tabular}

An ICC greater than 0.75 indicated good agreement

score $[r=0.602(95 \%$ CI $0.371-0.762) ; P=0.000]$, the groups of $\% \Delta \mathrm{V}$ moderately correlated with the Becker score $[r=0.416$ (95\% CI 0.136-0.635); $P=0.005]$, and the groups of WHO [ $r=0.075$ (95\% CI -0.227 to 0.364$) ; P=0.627]$, RECIST [ $r=0.270(95 \% \mathrm{CI}-0.0295$ to 0.525$) ; P=0.077$ ] and Choi criteria $[r=0.238$ (95\% CI -0.0637 to 0.499$)$; $P=0.120]$ showed no correlation with the Becker score (Table 3).

For the two readers, the mean time needed for the baseline examination was $12.3 \pm 3.2 \mathrm{~min}$ per patient, and the mean time needed for the follow-up examination was $11.5 \pm 2.9$ min per patient.

\section{Progression-free survival time}

Until December 2016, the mean follow-up time for all patients was $16 \pm 10$ months (range 4-49 months). During the observation period, 25 of 44 patients $(56.8 \%)$ experienced tumor recurrence or death.

According to the WHO, RECIST criteria, and $\% \Delta \mathrm{V}$, the difference in the PFS time between the responders and nonresponders was not significant $(P=0.513, P=0.108$, and $P=0.099$, respectively, by the log-rank test). In addition, the Choi and \% $\Delta$ TIU-p groups between the responders and nonresponders provided good correlation of PFS time $(P=0.013$ and $P=0.001$, respectively) (Table 3; Fig. 3d, e). According to the Choi group, the $75 \%$ PFS times of responders and non-responders were $11 \pm 2.4$ months and $7 \pm 1.9$ months, respectively. In the $\% \Delta$ TIU-p group, the $75 \%$ PFS times of responders and non-responders were $14 \pm 3.3$ months and $7 \pm 1.1$ months, respectively.

\section{Radiation dose}

The mean CTDIvol values at baseline and the followup examination for each patient were $24.8 \pm 3.4 \mathrm{mGy}$ and $25.3 \pm 3.1 \mathrm{mGy}$, respectively. The mean DLP values per patient at the two examinations were $831.2 \pm 213.6$ $\mathrm{mGy} \cdot \mathrm{cm}$ and $843.5 \pm 225.8 \mathrm{mGy} \cdot \mathrm{cm}$, respectively. The mean SSDE values per patient at the two examinations were $32.7 \pm 5.2 \mathrm{mGy}$ and $34.1 \pm 6.3 \mathrm{mGy}$, respectively.

\section{Discussion}

The results of this study demonstrated that the $\% \Delta$ TIU-p was strongly correlated to the histopathological grade of gastric cancer after neoadjuvant chemotherapy. Based on the $63.87 \%$ optimal cut-off values for the TIU-p reduction, the sensitivity and specificity to predict the histopathological response after neoadjuvant chemotherapy were high. In addition to Choi criteria, the $\% \Delta$ TIU-p could be another valuable predictive parameter of the PFS time for patients with gastric cancer after neoadjuvant chemotherapy.

Histologic changes in gastric carcinoma after chemotherapy include decreased tumor cell density, mucus lakes, fibrosis and chronic inflammatory infiltrates (Kiyabu et al. 2015). Cytotoxic chemotherapeutic agents can damage vascular endothelial cells and tumor cells, while the antiangiogenic targeting therapy can target the vascular supply of tumors (Thian et al. 2014a). Both can decrease the capacity of the vascular bed and reduce the blood content in tissues (Zhang et al. 2015). The iodinated contrast medium reaches the lesion primarily via blood perfusion from the viable tumor, blood volume and capillary permeability, and it consequently reflects this functional information about the tumor (Miles 1999). The damage caused by chemotherapy is characterized by the change in the contrast medium intake on CT images. Therefore, the IU derived from iodinated attenuation maps, which allows the calculation of iodine density, is assumed to be related to the accumulation of contrast medium and to indirectly reflect vital tumor burden (Dai et al. 2013; Uhrig et al. 2015).

The tumor volume allows for a more accurate quantification and can detect even small changes in the tumor lesion. Therefore, this approach may be an alternative promising strategy to assess the chemotherapy response based on morphology. Furthermore, this result was consistent with other studies (Murono et al. 2014; Rothe et al. 2013; Sang et al. 2009). However, the tumor burden cannot be clearly defined due to a complex and irregular tumor wall. Thus, the repeatability of the volume measurement between two readers was lower than that of the TIU measurement in our study. Compared with the $V$, the TIU covers not only the morphological but also the functional aspects of the entire lesion to quantitatively reflect the vital tumor cell burden. Thus, 
(a)

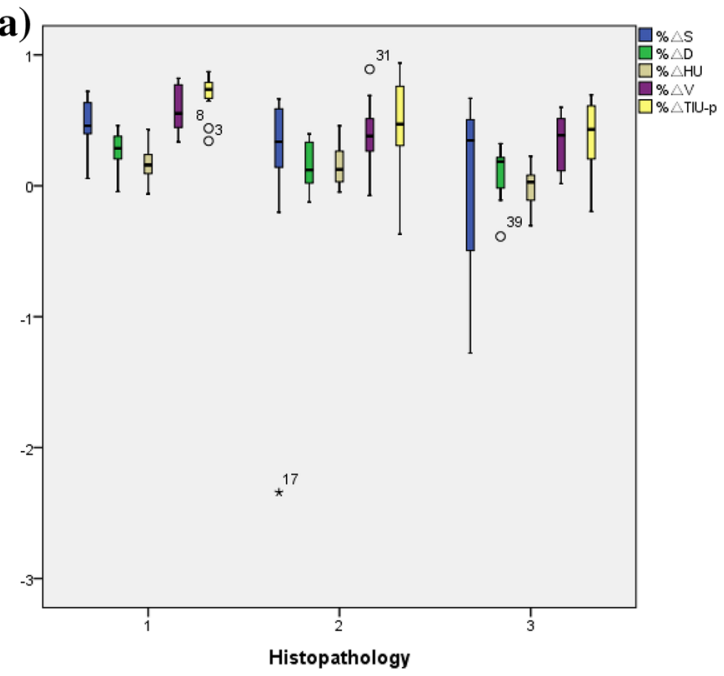

(c)

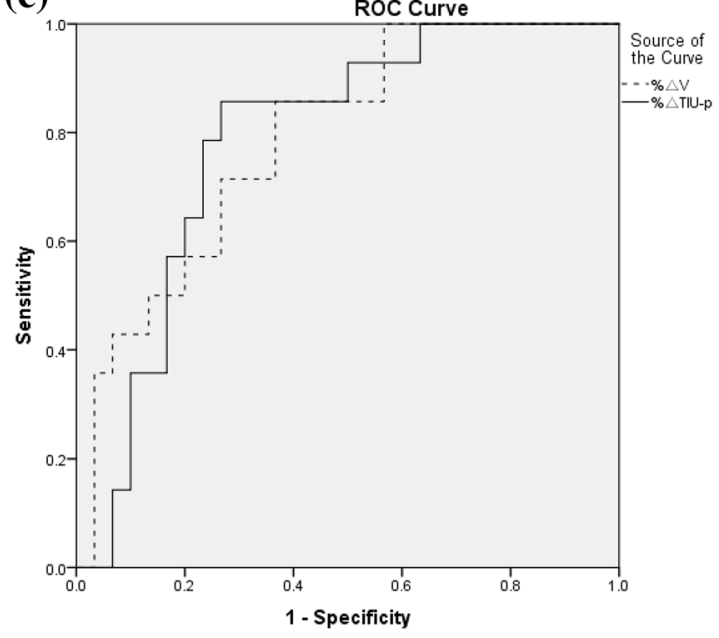

(e)

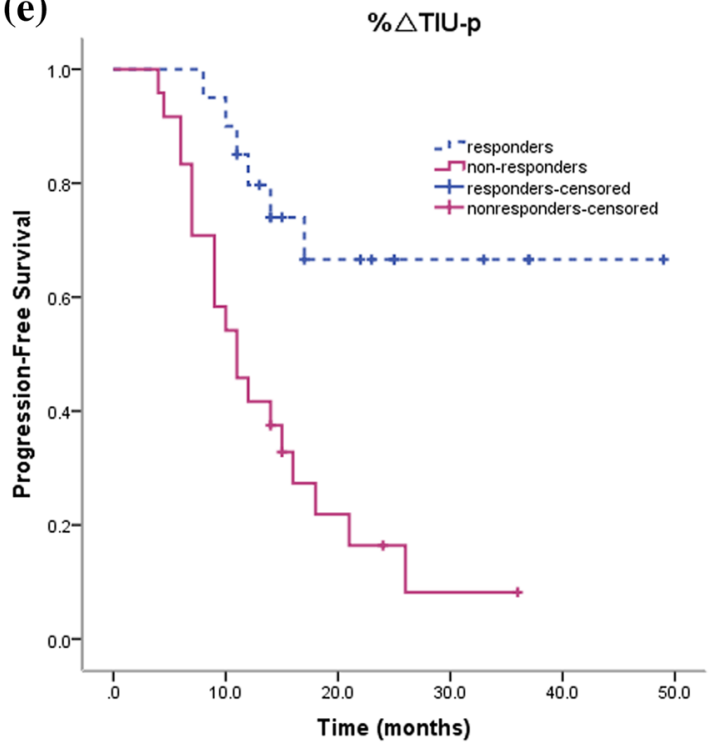

(b)

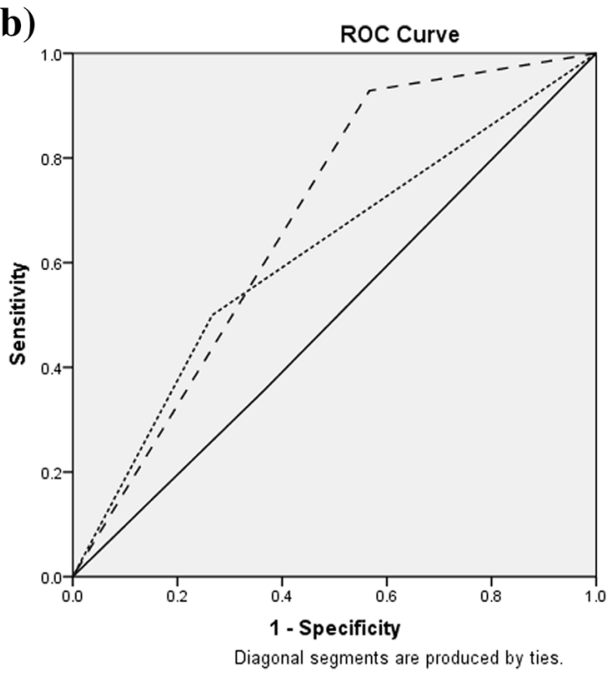

(d)

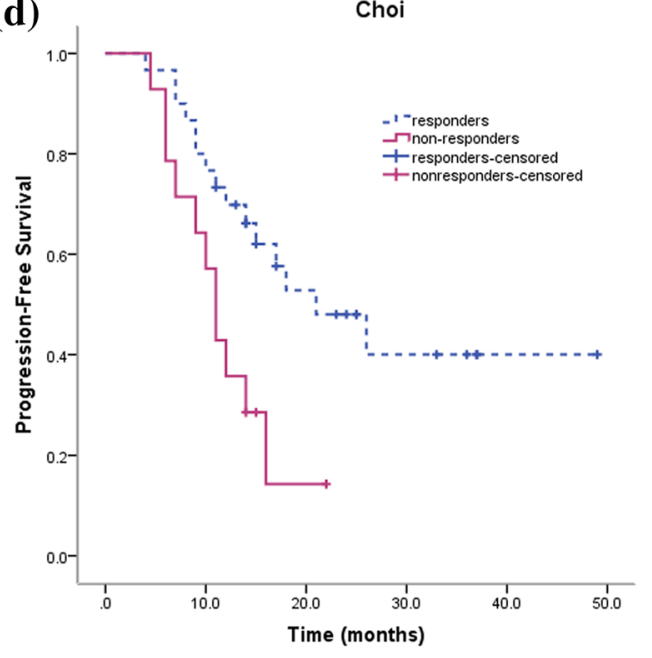


4Fig. 3 a The distribution of different parameters in different histopathologies; graphs, respectively, show ROCs to assess performance of WHO, RECIST, Choi criteria $\mathbf{b}$ and $\% \Delta V, \% \Delta$ TIU-p criteria $\mathbf{c}$ in discriminating responders and non-responders on the basis of pathologic grades; Kaplan-Meier plots for PFS of patients with advanced gastric cancer grouped by category: responders and non-responders as classified according to Choi criteria $\mathbf{d}$ and $\% \Delta \mathrm{TIU}-\mathrm{p}(\mathbf{e})$

the TIU is not affected by slight changes in the border. Our results are somewhat consistent with those of Tang et al., who showed that a change in the iodine concentration is related to the pathological regression of gastric cancer after neoadjuvant chemotherapy (Tang et al. 2015). Specifically, they obtained their data on the bi-dimensional planes rather than in the three-dimensional (3D) mode that was examined in our study. TIU was more accurate in reflecting overall metabolism of the primary tumor, as proven by Uhrig et al. in the evaluation of targeted therapy for melanoma metastases (Uhrig et al. 2013). Therefore, the TIU is a promising new functional assessment parameter to determine the effect of neoadjuvant chemotherapy for the treatment of gastric carcinoma and is assumed to reflect the vital tumor burden.

Defined by a bi-dimensional or uni-dimensional measurement, changes in the WHO, RECIST, and Choi criteria did not significantly correlate with pathological regression in our study $(P>0.05)$. Similar to our findings, previous studies have suggested that the use of different tumor response criteria may result in different assessments of treatment effect (Ahn et al. 2008; Apfaltrer et al. 2012; Park et al. 2003). Therefore, the traditional WHO, RECIST and Choi criteria cannot accurately evaluate the response to neoadjuvant chemotherapy of locally advanced gastric cancer or cancer in other irregularly shaped organs.
Table 2 Receiver operating characteristic analysis of the different criteria and parameters

\begin{tabular}{|c|c|c|c|c|c|}
\hline & WHO & RECIST & Choi & $\% \Delta V$ & $\% \Delta$ TIU-p \\
\hline AUC & $\begin{array}{l}0.495 \\
{[0.310} \\
0.680]\end{array}$ & $0.617[0.433,0.800]$ & $\begin{array}{c}0.681 \\
{[0.521} \\
0.840]\end{array}$ & $0.788[0.652,0.924]$ & $0.786[0.648,0.923]$ \\
\hline$P$ value & 0.960 & 0.217 & 0.055 & $0.002 *$ & $0.002^{*}$ \\
\hline Cut-off value & - & - & - & $\geq 43.34 \%$ & $\geq 63.87 \%$ \\
\hline Sensitivity & $35.7 \%$ & $50.0 \%$ & $92.9 \%$ & $85.7 \%$ & $85.7 \%$ \\
\hline Specificity & $63.3 \%$ & $73.3 \%$ & $43.3 \%$ & $63.3 \%$ & $73.3 \%$ \\
\hline Accuracy & $54.5 \%$ & $65.9 \%$ & $59.1 \%$ & $70.5 \%$ & $77.3 \%$ \\
\hline
\end{tabular}

$P$ value determined with receiver operating characteristic analysis

$* P<0.05$

\begin{tabular}{|c|c|c|c|c|c|c|c|}
\hline \multirow[t]{2}{*}{ Groups } & \multirow{2}{*}{$\begin{array}{l}\text { No. of } \\
\text { patients in } \\
\text { study }\end{array}$} & \multicolumn{3}{|c|}{ Histopathology } & \multirow{2}{*}{$\begin{array}{l}\text { Correlation } \\
\text { coefficient } \\
(\mathrm{r})^{\mathrm{a}}\end{array}$} & \multirow{2}{*}{$\begin{array}{l}P \text { values for } \\
\text { Spearman }^{\text {a }}\end{array}$} & \multirow{2}{*}{$\begin{array}{l}P \text { values } \\
\text { for survival } \\
\text { analysis }^{\mathrm{b}}\end{array}$} \\
\hline & & $\begin{array}{l}\text { Grade } 1 \\
(n=14)\end{array}$ & $\begin{array}{l}\text { Grade } 2 \\
(n=18)\end{array}$ & $\begin{array}{l}\text { Grade } 3 \\
(n=12)\end{array}$ & & & \\
\hline WHO criteria & & & & & 0.075 & 0.627 & 0.513 \\
\hline Responder & 16 & 5 & 8 & 3 & & & \\
\hline Non-responder & 28 & 9 & 10 & 9 & & & \\
\hline RECIST criteria & & & & & 0.270 & 0.077 & 0.108 \\
\hline Responder & 15 & 7 & 6 & 2 & & & \\
\hline Non-responder & 29 & 7 & 12 & 10 & & & \\
\hline Choi criteria & & & & & 0.238 & 0.120 & 0.013 \\
\hline Responder & 30 & 13 & 9 & 8 & & & \\
\hline Non-responder & 14 & 1 & 9 & 4 & & & \\
\hline V group & & & & & 0.416 & 0.005 & 0.099 \\
\hline Responder & 23 & 12 & 7 & 4 & & & \\
\hline Non-responder & 21 & 2 & 11 & 8 & & & \\
\hline TIU-p group & & & & & 0.602 & 0.000 & 0.001 \\
\hline Responder & 20 & 12 & 7 & 1 & & & \\
\hline Non-responder & 24 & 2 & 11 & 11 & & & \\
\hline
\end{tabular}

${ }^{\mathrm{a}} r$ Determined with correlation coefficient for Spearman correlation analysis

${ }^{\mathrm{b}} P$ value for survival analysis determined with the log-rank test
Table 3 Stratification of patients by histopathologic grade for different groups 

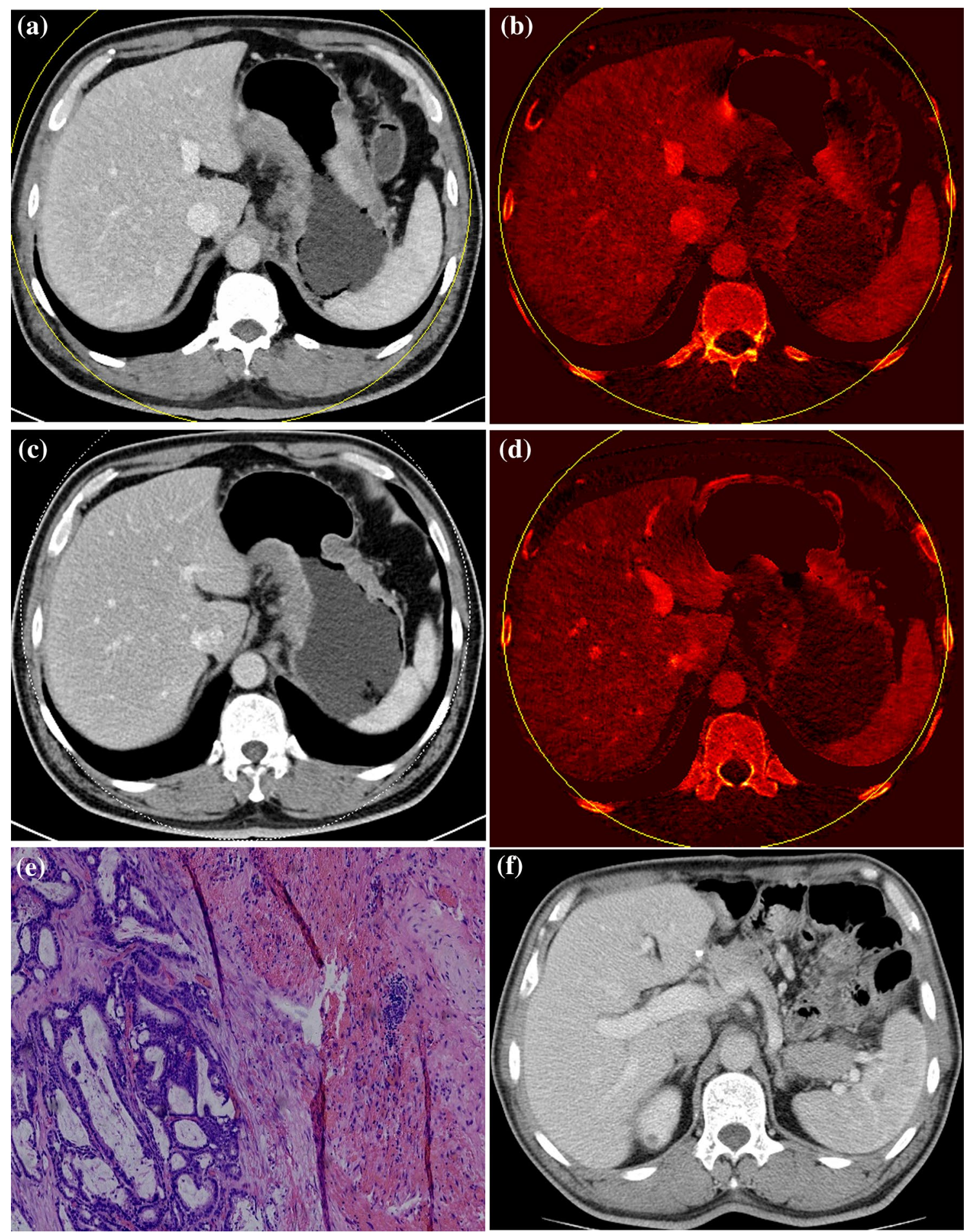

Fig. 4 Baseline CT image (a, b) and follow-up CT image (c, d) of a patient (male, 51 years) after three cycles of chemotherapy $(\% \Delta \mathrm{S}$ : $-21.44 \%, \% \Delta \mathrm{D}:-26.88 \%, \% \Delta \mathrm{HU}:-14.66 \%, \% \Delta \mathrm{V}:-39.74 \%$, $\% \Delta$ TIU-p: $-60.09 \%$ ). According to the Choi criteria, this patient was classified as a responder, but according to other criteria, he was

classified as a non-responder. Finally, the pathology of this patient (e) was grade 2, minimal tumor regression (40\% residual tumor per tumor bed with intratumoral hemorrhage). Furthermore, this patient was found to have spleen metastasis after 15 months (f) 
Although the IU of DECT has been shown to be able to evaluate the responses of various tumors to therapy in previous studies (Apfaltrer et al. 2012; Dai et al. 2013; Uhrig et al. 2013), survival analyses have not been carried out before. Our results showed that the PFS time significantly differed between the responder and non-responder groups based on the \% $\%$ TIU-p and Choi criteria. There were different perspectives for Choi criteria to predict the survival time based on the previous studies (Krajewski et al. 2011; Liu et al. 2012; Thian et al. 2014b). Liu et al. (2012) thought that Choi criteria may be helpful to predict PFS and OS in AGC after chemotherapy. However, there are literatures (Krajewski et al. 2011; Thian et al. 2014b) which report that response according to Choi criteria was not significantly correlated with PFS or OS. In our study, 12 patients were inconsistently judged in the group $\% \Delta$ TIU-p and Choi group and 10 patients in the group $\% \Delta$ TIU-p matched the histological regression grade while 2 patients in the group Choi matched. The number of responders and non-responders by Choi criteria was very different (15:7), because of a wider range within the responder group compared with the RECIST guidelines. We speculate that this small number of nonresponders resulted in a bias for the PFS time. On the other hand, we analyzed PFS instead of the overall survival which is the most reliable endpoint in clinical studies, but it will only be available after a longer time than PFS. For example, considering the patient in Fig. 4 after three cycles of neoadjuvant chemotherapy $(\% \Delta \mathrm{S}:-21.44 \%, \% \Delta \mathrm{D}:-26.88 \%$, $\% \Delta \mathrm{HU}:-14.66 \%, \% \Delta \mathrm{V}:-39.74 \%, \% \Delta \mathrm{TIU}-\mathrm{p}:-60.09 \%)$, this patient was classified as a responder by Choi criteria, but he was classified as a non-responder according to $\% \Delta$ TIU-p. Finally, the surgical pathological finding of this patient was minimal tumor regression, and intratumoral hemorrhage was shown. The patient was identified with spleen metastasis after 15 months. Therefore, the TIU more accurately reflects the PFS time than the Choi criteria does to some extent.

The present study has some limitations that warrant consideration. First, the retrospective selection of the optimal cut-off thresholds that maximized the sum of sensitivity and specificity is potentially susceptible to over-interpretation bias of the study results. Second, the small sample size and the follow-up time were not sufficiently long because some patients could not endure the side effects of chemotherapy, and the survival time of patients with gastric cancer is shorter. Thus, a larger investigation is needed to confirm our findings. Third, the aim of our study was to evaluate primary lesions and did not involve lymph node, and further studies should be designed to evaluate metastatic lymph nodes and other independent prognostic factors for gastric cancer. Fourth, the boundaries of small lesions may not be well-defined in larger sample sizes. Fortunately, lesions were measurable in all 44 patients; the minimum volume was $8.6 \mathrm{~cm}^{3}$, and the volume ranged from 8.6 to $290.1 \mathrm{~cm}^{3}$. Finally, the measurement time per patient was relatively long. Nevertheless, improvements in the software will overcome this problem.

In conclusion, the TIU-p can help to predict pathological regression of the primary lesion after neoadjuvant chemotherapy in patients with advanced gastric cancer, while the traditional WHO, RECIST and Choi criteria cannot accurately evaluate the pathological response. In addition, the $\% \Delta$ TIU-p could be one of the potentially valuable predictive parameters of the PFS time for patients with gastric cancer after neoadjuvant chemotherapy.

Funding This study was funded by Science Fund of the National Natural Science Foundation of China (NSFC) 81771789, U1532107.

\section{Compliance with ethical standards}

Conflict of interest The authors declare that they have no conflict of interest.

Ethical approval All procedures performed in studies involving human participants were in accordance with the ethical standards of the institutional and/or national research committee and with the 1964 Helsinki declaration and its later amendments or comparable ethical standards.

Informed consent Informed consent was obtained from all individual participants included in the study.

Open Access This article is distributed under the terms of the Creative Commons Attribution 4.0 International License (http://creativeco mmons.org/licenses/by/4.0/), which permits unrestricted use, distribution, and reproduction in any medium, provided you give appropriate credit to the original author(s) and the source, provide a link to the Creative Commons license, and indicate if changes were made.

\section{References}

Ahn SH, Garewal HS, Dragovich T (2008) Discrepancy in the assessment of tumor response in patients with pancreatic cancer: WHO versus RECIST criteria. J Buon 13:359-362

Apfaltrer P et al (2012) Contrast-enhanced dual-energy CT of gastrointestinal stromal tumors: is iodine-related attenuation a potential indicator of tumor response? Invest Radiol 47:65-70

Becker K et al (2003) Histomorphology and grading of regression in gastric carcinoma treated with. Neoadjuvant Chemother Cancer 98:1521-1530

Benjamin RS et al (2007) We should desist using RECIST, at least in GIST. J Clin Oncol 25:1760-1764

Bichev D et al (2015) High impact of histopathological remission for prognosis after perioperative chemotherapy with ECF and ECFLike Regimens for gastric and. Gastroesophageal Adenocarcinoma 89:95-102

Chen R, Zhou X, Liu J, Huang G (2016) Relationship between 18FFDG PET-CT scans and HER2 expression in gastric cancer. $J$ Nucl Med 57:1040

Choi H (2008) Response evaluation of gastrointestinal stromal tumors Oncologist 13(Suppl 2):4

Christner JA, Braun NN, Jacobsen MC, Carter RE, Kofler JM, Mccollough CH (2013) Size-specific dose estimates for adult patients at CT of the torso International. J Med Radiol 265:841 
Dai X, Schlemmer HP, Schmidt B, Höh K, Xu K, Ganten TM, Ganten MK (2013) Quantitative therapy response assessment by volumetric iodine-uptake measurement: initial experience in patients with advanced hepatocellular carcinoma treated with sorafenib European. J Radiol 82:327

Eisenhauer EA et al (2009) New response evaluation criteria in solid TUMORs: revised RECIST guideline (version 1.1) Gan to Kagaku Ryoho. Cancer Chemother 36:2495-2501

Giganti F et al (2015) Response to chemotherapy in gastric adenocarcinoma with diffusion-weighted MRI and (18) F-FDG-PET/ CT. correlation of apparent diffusion coefficient and partial volume corrected standardized uptake value with histological tumor regression grade. J Magn Reson Imaging 40:1147-1157

Grady L (2006) Random walks for image segmentation. IEEE Trans Pattern Anal Mach Intell 28:1768-1783

Kiyabu M, Leichman L, Chandrasoma P (2015) Effects of preoperative chemotherapy on gastric adenocarcinomas. A morphologic study of 25 cases. Cancer 70:2239-2245

Krajewski KM et al (2011) Comparison of four early posttherapy imaging changes (EPTIC; RECIST 1.0, tumor shrinkage, computed tomography tumor density, Choi criteria) in assessing outcome to vascular endothelial growth factor-targeted therapy in patients with advanced renal cell carcinoma. Eur Urol 59:856-862. https ://doi.org/10.1016/j.eururo.2011.01.038

Landis JR, Koch GG (1977) The measurement of observer agreement for categorical data. Biometrics 33:159-174

Lee JW, Jo K, Cho A, Noh SH, Lee JD, Yun M (2015) Relationship between 18F-FDG uptake on PET and recurrence patterns after curative surgical resection in patients with advanced gastric cancer. J Nucl Med 56:1494-1500

Liu K, Li G, Fan C, Zhou C, Li J (2012) Adapted Choi response criteria for prediction of clinical outcome in locally advanced gastric cancer patients following preoperative chemotherapy. Acta Radiol 53:127

Miles KA (1999) Tumour angiogenesis and its relation to contrast enhancement on computed tomography: a review European. J Radiol 30:198-205

Motzer RJ et al (2006) Activity of SU11248, a multitargeted inhibitor of vascular endothelial growth factor receptor and platelet-derived growth factor receptor, in patients with metastatic renal cell carcinoma. J Clin Oncol Off J Am Soc Clin Oncol 24:16

Murono K et al (2014) Barium enema and CT volumetry for predicting pathologic response to preoperative chemoradiotherapy in rectal cancer patients. Disease Colon Rectum 57:715-724

Ott K et al (2003) Prediction of response to preoperative chemotherapy in gastric carcinoma by metabolic imaging: results of a prospective trial. J Clin Oncol 21:4604-4610

Park JO et al (2003) Measuring response in solid tumors: comparison of RECIST and WHO response criteria Japanese. J Clin Oncol 33:533-537

Putter H (2006) Perioperative chemotherapy versus surgery alone for resectable gastroesophageal cancer. New Engl J Med 355:11

Rothe JH et al (2013) Size determination and response assessment of liver metastases with computed tomography-comparison of RECIST and volumetric algorithms European. J Radiol 82:1831-1839

Sánchez-Gracián CD, Pernas RO, López CT, Armentia ES, Liste AV, Caamaño MV, Fuente GTDL (2015) Quantitative myocardial perfusion with stress dual-energy CT: iodine concentration differences between normal and ischemic or necrotic myocardium. Initial Exp Eur Radiol 26:3199-3207

Sang ML et al (2009) Usefulness of CT volumetry for primary gastric lesions in predicting pathologic response to neoadjuvant chemotherapy in advanced gastric cancer. Abdom Imaging 34:430-440

Shi C et al (2016) Gastric cancer: preoperative TNM staging with individually adjusted computed tomography scanning phase. J Comput Assist Tomogr 40:160-166. https://doi.org/10.1097/ RCT.0000000000000339

Staquet M (1981) Reporting results of cancer treatment. Cancer 47:207-214

Stiller W et al (2015) Correlation of quantitative dual-energy computed tomography iodine maps and abdominal computed tomography perfusion measurements: are single-acquisition dual-energy computed tomography iodine maps more than a reduced-dose surrogate of conventional. Comput Investig Radiol 50:703

Tang L et al (2015) Evaluating the response of gastric carcinomas to neoadjuvant chemotherapy using iodine concentration on spectral CT: a comparison with pathological regression. Clin Radiol 70:1198-1204

Thian Y, Gutzeit A, Koh DM, Fisher R, Lote H, Larkin J, Sohaib A (2014a) Revised Choi imaging criteria correlate with clinical outcomes in patients with metastatic renal cell carcinoma treated with sunitinib. Radiology 273:452-461. https://doi.org/10.1148/ radiol.14132702

Thian Y, Gutzeit A, Koh DM, Fisher R, Lote H, Larkin J, Sohaib A (2014b) Revised choi imaging criteria correlate with clinical outcomes in patients with metastatic renal cell carcinoma treated with sunitinib. Radiology 273:452-461

Uhrig M, Sedlmair M, Schlemmer HP, Hassel JC, Ganten M (2013) Monitoring targeted therapy using dual-energy CT: semi-automatic RECIST plus supplementary functional information by quantifying iodine uptake of melanoma metastases. Cancer Imaging 13:306-313

Uhrig M, Simons D, Ganten MK, Hassel JC, Schlemmer HP (2015) Histogram analysis of iodine maps from dual energy computed tomography for monitoring targeted therapy of melanoma patients. Fut Oncol 11:591-606

Ychou M et al (2011) Perioperative chemotherapy compared with surgery alone for resectable gastroesophageal adenocarcinoma: an FNCLCC and FFCD multicenter phase III trial. J Clin Oncol Off J Am Soc Clin Oncol 29:1715-1721

Yeh BM, Shepherd JA, Zhen JW, Hui ST, Hartman R, Prevrhal S (2009) Dual energy and low kVp CT in the Abdomen Ajr American. J Roentgenol 193:47

Zhang L, Tong Y, Zhang X, Pan M, Chen S (2015) Arsenic sulfide combined with JQ1, chemotherapy agents, or celecoxib inhibit gastric and colon cancer cell growth. Drug Des Dev Therap 9:5851-5862 